Europhysics Letters

PREPRINT

\title{
Multi-Agent Complex Systems and Many-Body Physics
}

\author{
Neil F. Johnson ${ }^{1}$, David M.D. Smith ${ }^{2}$ and Pak Ming Hui ${ }^{3}$ \\ 1 Physics Department, Oxford University, Oxford, OX1 3PU, UK \\ 2 Mathematics Department, Oxford University, Oxford, OX1 2EL, UK \\ 3 Department of Physics, Chinese University of Hong Kong, Hong Kong \\ PACS. nn.mm. $x x-87.23 . G e$. \\ PACS. nn.mm. $\mathrm{xx}-73.21 .-\mathrm{b}$. \\ PACS. nn.mm.xx - 87.23.-n.
}

\begin{abstract}
Multi-agent complex systems comprising populations of decision-making particles, have many potential applications across the biological, informational and social sciences. We show that the time-averaged dynamics in such systems bear a striking resemblance to conventional many-body physics. For the specific example of the Minority Game, this analogy enables us to obtain analytic expressions which are in excellent agreement with numerical simulations.
\end{abstract}

Multi-agent simulations are currently being used to study the dynamical behavior within a wide variety of Complex Systems [1]. Within these simulations, $N$ decision-making particles or agents (e.g. commuters, traders, computer programs [2-4]) repeatedly compete with each other for some limited global resource (e.g. road space, best buy/sell price, processing time) using sets of rules which may differ between agents and may change in time. The population is therefore competitive, heterogeneous and adaptive. A simple version of such a scenario, which has generated more than one hundred papers since 1998, is the Minority Game (MG) of Challet and Zhang [2-9].

Here we show that the time-averaged dynamics of such multi-agent systems - in particular, their $n$-point correlation functions - can be interpreted as a generalization of conventional many-body physics [10]. We also show that these correlation functions can be evaluated accurately if one regroups the agents into clusters of like particles (i.e. crowds) and their anticorrelated mirror-images (i.e. anticrowds). When applied to the MG, this approach yields a set of analytic results which are in excellent agreement with the numerical findings of Savit et al. (see Fig. 1) [6]. Although there have been many other MG theories proposed to date [5], none of these provides an analytic description of this Savit-curve [6] (Fig. 1) over the entire parameter space.

Figure 2 shows a generic setup of such a multi-agent system. The Minority Game represents a specific example of this setup, in which the identity of the minority group provides the global outcome - however, what follows does not depend specifically on such a minority rule. There are $N$ agents (e.g. commuters) who repeatedly decide between two actions at each timestep $t$ (e.g. $+1 /-1 \equiv$ take route A/B) using their individual $S$ strategies. The agents have access to some common information $\mu(t)$. For example, Fig. 2 shows $\mu(t)$ to be the past few outcomes

(C) EDP Sciences 


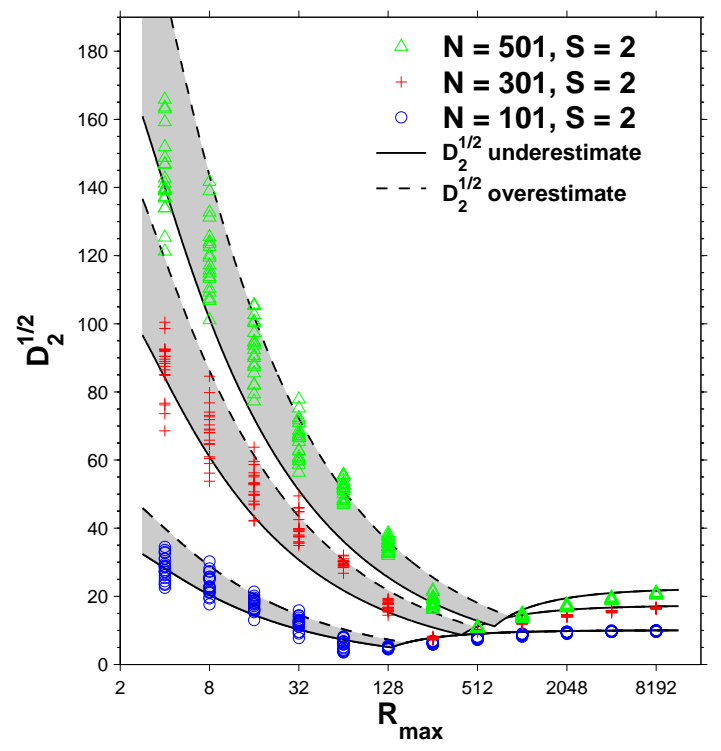

Fig. 1 - Results for the standard deviation of fluctuations $D_{2}^{1 / 2}$ in the Minority Game. Numerical results correspond to 20 different runs at each $N$ and $R_{\max }$. The theoretical curves are generated using the analytic expressions in the text. The shaded area bounded by the upper and lower curves shows our theoretical prediction of the numerical spread for a given $N$. In line with the original numerical results of Ref. [6], we have chosen successive $R_{\max }$ tick-values to increase by a factor of 4 .

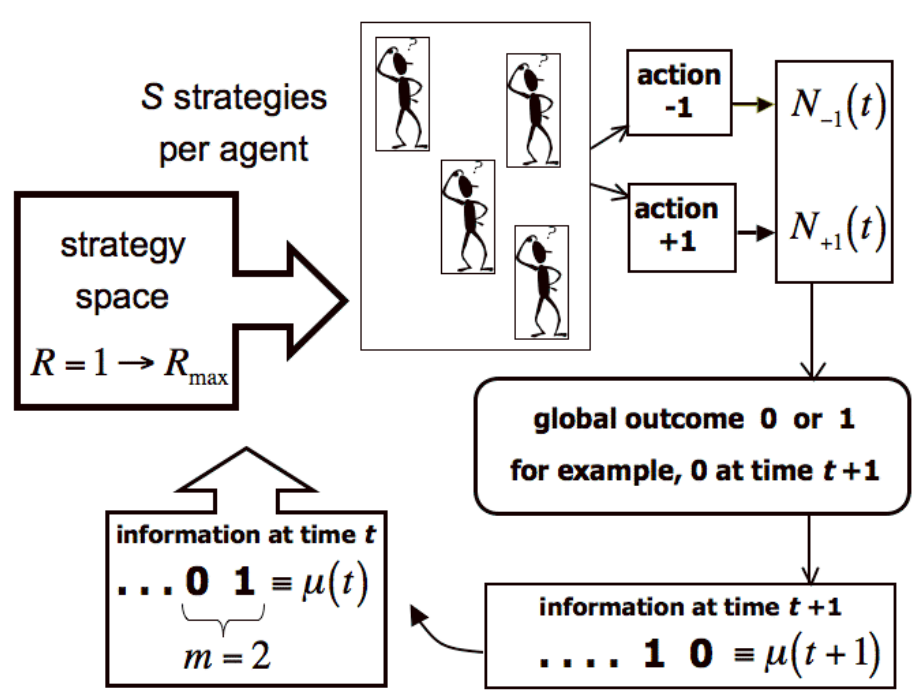

Fig. 2 - General setup: At timestep $t$, each agent decides between action -1 and action +1 based on the predictions of the $S$ strategies that he possesses. $N_{-1}[t]$ agents choose -1 , and $N_{+1}[t]$ choose +1 . A global outcome 0 or 1 is assigned depending on the rules of the game (e.g. minority group wins). Strategies are rewarded/penalized one virtual point according to whether their predicted action would have been a winning/losing action. 


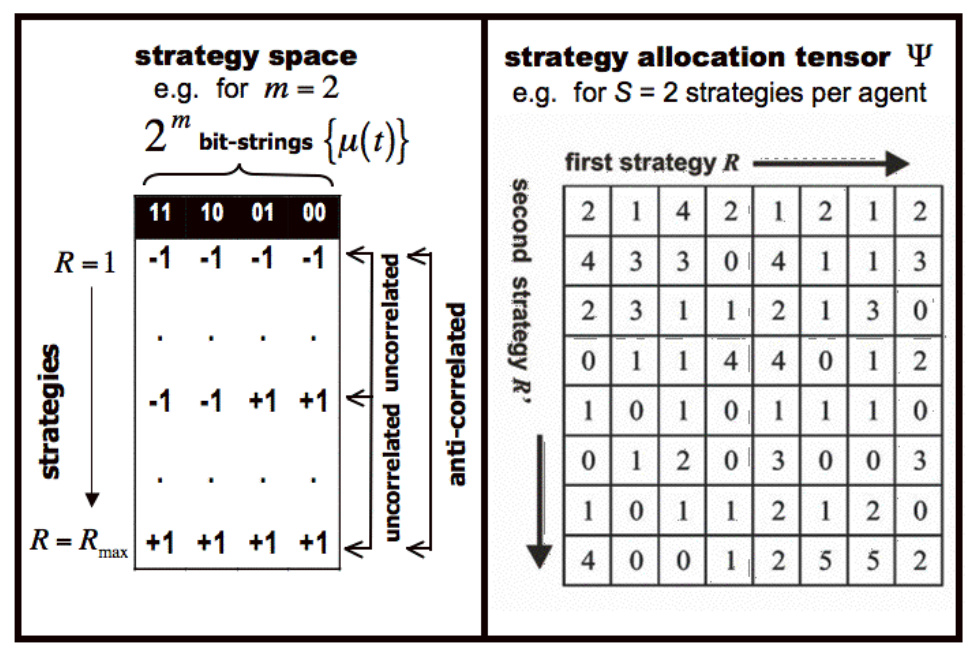

Fig. 3 - Left: strategy space for $m=2$, together with some example strategies. The reduced strategy space contains $2.2^{m}=2 P$ strategies with specific correlations (i.e. fully correlated, uncorrelated or anti-correlated [4,5]). Right: strategy allocation tensor $\Psi$ in the case of $S=2$ strategies per agent. Each square shows the number of agents who were assigned strategy $R$ and then $R^{\prime}$.

of the system where 0 or 1 could represent route A or B being the least crowded, respectively. Hence $\mu(t)$ is, in the setup of Fig. 2, a binary string of outcomes. Each strategy, labelled $R$, comprises a particular action $a_{R}^{\mu(t)}= \pm 1$ for each $\mu(t) \in\{\mu(t)\}$, and the set of all possible strategies constitutes a strategy space $\Theta \equiv\{R\}$. Figure 3 (left) gives an example of such a strategy space in the case that each agent has information $\mu(t)$ corresponding to the most recent $m=2$ outcomes. Each agent has some subset $S$ of these strategies, hence the strategy allocation among agents can be described in terms of a rank- $S$ tensor $\Psi$ [9] where each entry gives the number of agents holding a particular combination of $S$ strategies. Figure 3 (right) gives an example of this strategy allocation matrix $\Psi$ for $S=2$. We assume $\Psi$ to be constant over the timescale for which time-averages are taken. A single $\Psi$ 'macrostate' corresponds to many possible 'microstates' describing the specific partitions of strategies among the $N$ agents [9]. To allow for large strategy spaces and large sets of global information, we can convert the strategies $\{R\}$ to their decimal equivalents and label them from $R=1 \rightarrow R_{\max }$, where $R_{\max }=2.2^{m}$, in order of increasing magnitude. Hence for the example strategies in Fig. 3, we can relabel strategy [ $-1-1-1-1]$ as $R=1,[-1-1+1+1]$ as $R=4$ and $[+1+1+1+1]$ as $R=R_{\max }=8$. Hence $R$ mimics a 'coordinate' in that if an agent is using a particular strategy $R$, the agent can be thought of as sitting on a line at coordinate $R$. For large strategy spaces, there will be many possible $R$-values - for example, $m \geq 3$ means that there are $2.2^{m} \geq 16$ strategies in the reduced strategy space of Fig. 3. The line of ordered strategies, and hence allowed $R$-values, will therefore appear quite dense. Given this, we will take the liberty of writing down sums over $R$ as integrals over $R$ in the rest of this paper.

We denote the number of agents choosing -1 (e.g. take route $\mathrm{A}$, or buy a given stock) and +1 (e.g. take route $\mathrm{B}$, or sell a given stock) as $N_{-1}(t)$ and $N_{+1}(t)$ respectively. Many macroscopic quantities of interest can be written in terms of $N_{-1}(t)$ and $N_{+1}(t)$. For example, 
the excess traffic on route $\mathrm{A}$ as compared to route $\mathrm{B}$, or the excess number of buyers over sellers of a given stock and hence its price-change at time $t$, can be written as $D(t)=N_{+1}(t)-N_{-1}(t)$. Hence we will focus here on $D(t)$, which is given by:

$$
D(t) \equiv \int_{R=1}^{R_{\max }} d R a_{R}^{\mu(t)} n \frac{S}{R}(t) ; \Psi,
$$

where $n \frac{S}{R}(t) ; \Psi$ is the number of agents using strategy $R$ at time $t$, and $\underline{S}(t)$ is the current scorevector denoting the past performance of each strategy [9]. In particular, the element $S_{R}(t)$ represents the net number of successful predictions of strategy $R$ up to time $t$. Therefore, the combination of $\underline{S}(t), \Psi$ and the game rule (e.g. use strategy with best or second-best performance to date) defines $n \frac{S}{R}(t) ; \Psi$. The action $a_{R}^{\mu(t)}= \pm 1$ is determined uniquely by $\mu(t)$.

In conventional many-body physics, we are typically interested in the following statistical properties of macroscopic measurable quantities such as $D(t)$ : (i) the moments of the probability distribution function (PDF) of $D(t)$ (e.g. mean, variance, kurtosis) and (ii) the correlation functions that are products of $D(t)$ at different times $t_{1}=t, t_{2}=t+\tau, t_{3}=t+\tau^{\prime}$ etc. (e.g. autocorrelation). Numerical multi-agent simulations typically average over time $t$ and then over configurations $\{\Psi\}$. A general expression to generate all such functions, is therefore

$$
\begin{aligned}
& D_{P}^{\left(\tau, \tau^{\prime}, \tau^{\prime \prime}, \ldots\right)} \equiv\left\langle\left\langle D\left(t_{1}\right) D\left(t_{2}\right) \ldots D\left(t_{P}\right)\right\rangle_{t}\right\rangle_{\Psi}
\end{aligned}
$$

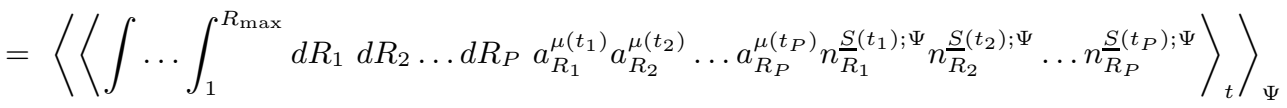

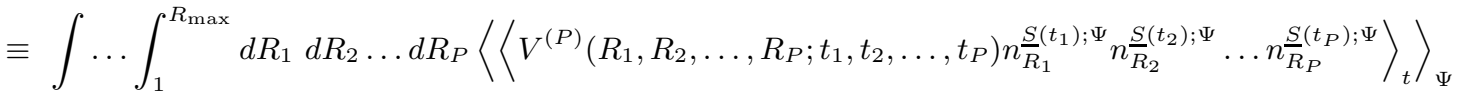

where

$$
V^{(P)}\left(R_{1}, R_{2} \ldots, R_{P} ; t_{1}, t_{2} \ldots, t_{P}\right) \equiv a_{R_{1}}^{\mu\left(t_{1}\right)} a_{R_{2}}^{\mu\left(t_{2}\right)} \ldots a_{R_{P}}^{\mu\left(t_{P}\right)}
$$

resembles a time-dependent, non-translationally invariant, $p$-body interaction potential in $\mathbf{R} \equiv$

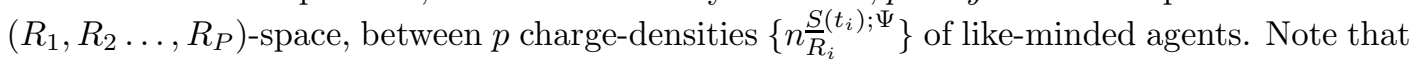
each charge-density $n \frac{S}{R_{i}}\left(t_{i}\right) ; \Psi$ now possesses internal degrees of freedom determined by $\underline{S}(t)$ and $\Psi$. Since $\left\{n \frac{S}{R_{i}}\left(t_{i}\right) ; \Psi\right\}$ are determined by the game's rules, Eq. (2) can be applied to any multiagent game, not just the MG. We focus here on moments of the PDF of $D(t)$ where $\left\{t_{i}\right\} \equiv t$ and hence $\{\tau\}=0$. Discussion of temporal correlation functions such as the autocorrelation $D_{2}^{(\tau)}$ will be reported elsewhere. We consider explicitly the variance $D_{2}$ to demonstrate the approach, noting that higher-order moments such as $D_{4}$ (i.e. kurtosis) which classify the nonGaussianity of the PDF, can be treated in a similar way. The potential $V^{(P)}$ is insensitive to the configuration-average over $\{\Psi\}$, hence the mean is given by [11]:

$$
D_{1}=\int_{R=1}^{R_{\max }} d R\left\langle V^{(1)}(R ; t)\left\langle n \frac{S}{R}(t) ; \Psi\right\rangle_{\Psi}\right\rangle_{t} .
$$

If the game's output is unbiased, the averages yield $D_{1}=0$. This condition is not necessary one can simply subtract $D_{1}^{2}$ from the right hand side of the expression for $D_{2}$ below - however we will take $D_{1}=0$ for clarity. The variance $D_{2}$ measures the fluctuations of $D(t)$ about its average value:

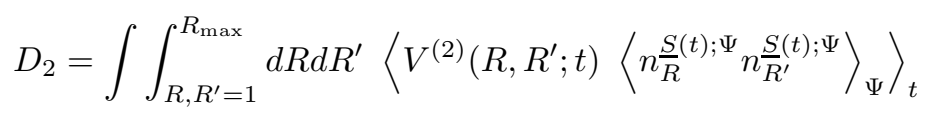


where $V^{(2)}\left(R, R^{\prime} ; t\right) \equiv a_{R}^{\mu(t)} a_{R^{\prime}}^{\mu(t)}$ acts like a time-dependent, non-translationally invariant, two-body interaction potential in $\left(R, R^{\prime}\right)$-space.

These effective charge-densities and potential fluctuate in time. It is reasonable to assume

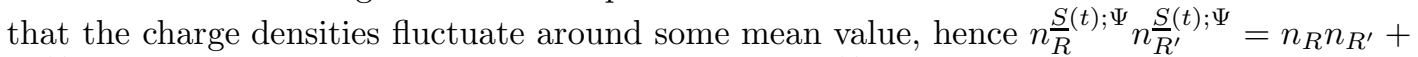

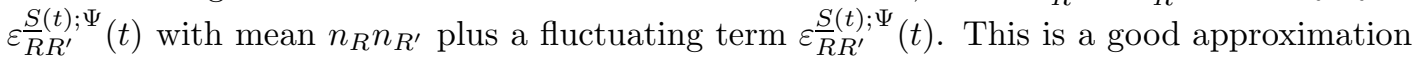
if we take $R$ to be a popularity-ranking (i.e. the $R$ th most popular strategy) or a strategyperformance ranking (i.e. the $R$ th best-performing strategy) since in these cases $n \frac{S}{R}(t)$; $\Psi$ will be reasonably constant [4]. For example, taking $R$ as a popularity-ranking implies $n \frac{S}{R}(t) ; \Psi \geq$

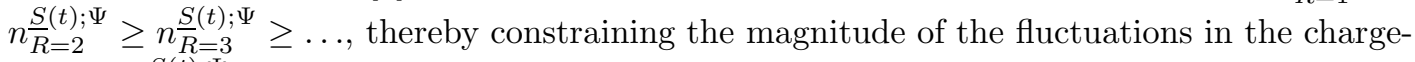
density $n \frac{S}{R}(t) ; \Psi$. Hence

$$
D_{2}=\iint_{R, R^{\prime}=1}^{R_{\max }} d R d R^{\prime}\left\langle V^{(2)}\left(R, R^{\prime} ; t\right)\left\langle n_{R} n_{R^{\prime}}+\varepsilon \frac{S}{R} R^{\prime}(t)(t)\right\rangle_{\Psi}\right\rangle_{t} .
$$

We will assume that $\varepsilon \frac{S}{R}(t) ; \Psi$ ( $(t)$ averages out to zero. In the presence of network connections between agents, there can be strong correlations between these noise terms $\varepsilon \frac{S}{R} R^{\prime}(t)$ and the time-dependence of $V^{(2)}\left(R, R^{\prime} ; t\right)$, implying that the averaging over $t$ should be carried out timestep-by-timestep [12]. For MG-like games without connections, the agents cannot suddenly access larger numbers of strategies and hence these correlations can be ignored. This gives

$$
D_{2}=\iint_{R, R^{\prime}=1}^{R_{\max }} d R d R^{\prime}\left\langle V^{(2)}\left(R, R^{\prime} ; t\right)\right\rangle_{t} n_{R} n_{R^{\prime}} .
$$

As in conventional many-body theory, the expectation value in Eq. (7) can be 'contracted' down by making use of the equal-time correlations between $\left\{a_{R}^{\mu(t)}\right\}$. As is well-known for MG-like games $[2,5,8]$, we can safely work in the so-called reduced strategy space which is constructed such that any pair $R$ and $R^{\prime}$ are either (i) fully correlated, i.e. $a_{R}^{\mu(t)}=a_{R^{\prime}}^{\mu(t)}$ for all $\mu(t)$; (ii) anti-correlated, i.e. $a_{R}^{\mu(t)}=-a_{R^{\prime}}^{\mu(t)}$ for all $\mu(t)$; (iii) uncorrelated, i.e. $a_{R}^{\mu(t)}=a_{R^{\prime}}^{\mu(t)}$ for half of $\{\mu(t)\}$ while $a_{R}^{\mu(t)}=-a_{R^{\prime}}^{\mu(t)}$ for the other half of $\{\mu(t)\}$. In other words, one can choose two subsets of the strategy space $\Theta$, i.e. $\Theta=U \oplus \bar{U}$, such that the strategies within $U$ are uncorrelated, the strategies within $\bar{U}$ are uncorrelated, the anticorrelated strategy of $R \in U$ appears in $\bar{U}$, and the anticorrelated strategy of $R \in \bar{U}$ appears in $U$. We can therefore break up the integrals in Eq. (7): (i) $R^{\prime} \equiv R$ (i.e. fully-correlated) hence $\frac{1}{\mu_{\max }} \int_{\mu=1}^{\mu_{\max }} d \mu a_{R}^{\mu} a_{R^{\prime}}^{\mu}=1$ and $\left\langle V^{(2)}\left(R, R^{\prime} ; t\right)\right\rangle_{t}=1$; (ii) $R^{\prime} \equiv \bar{R}$ (i.e. anticorrelated) which yields $\frac{1}{\mu_{\max }} \int_{\mu=1}^{\mu_{\max }} d \mu a_{R}^{\mu} a_{R^{\prime}}^{\mu}=$ -1 . If all possible global information values $\{\mu\}$ are visited reasonably equally over a long time-period, this implies $\left\langle V^{(2)}\left(R, R^{\prime} ; t\right)\right\rangle_{t}=-1$. For the MG, for example, $\{\mu\}$ corresponds to the $m$-bit histories which indeed are visited equally for small $m$. For large $m$, they are not visited equally for a given $\Psi$, but are when averaged over all $\Psi$. If, by contrast, we happened to be considering some general non-MG game where the $\mu$ 's occur with unequal probabilities $\rho_{\mu}$, even after averaging over all $\Psi$, one can simply redefine the strategy subsets $U$ and $\bar{U}$ to yield a generalized scalar product, i.e. $\frac{1}{\mu_{\max }} \int_{\mu=1}^{\mu_{\max }} d \mu a_{R}^{\mu} a_{R^{\prime}}^{\mu} \rho_{\mu}=-1$ (or 0 in case (iii)). (iii) $R^{\prime} \perp R$ (i.e. uncorrelated) which yields $\frac{1}{\mu_{\max }} \int_{\mu=1}^{\mu_{\max }} d \mu a_{R}^{\mu} a_{R^{\prime}}^{\mu}=0$ and hence $\left\langle V^{(2)}\left(R, R^{\prime} ; t\right)\right\rangle_{t}=0$. Hence

$$
D_{2}=\iint_{R, R^{\prime}=1}^{R_{\max }} d R d R^{\prime}\left\langle V^{(2)}\left(R, R^{\prime} ; t\right)\right\rangle_{t} n_{R} n_{R^{\prime}}=\int_{R=1}^{R_{\max }} d R\left(n_{R} n_{R}-n_{R} n_{\bar{R}}\right)
$$




$$
\begin{aligned}
& =\int_{R \in U} d R\left(n_{R} n_{R}-n_{R} n_{\bar{R}}+n_{\bar{R}} n_{\bar{R}}-n_{\bar{R}} n_{R}\right) \\
& =\int_{R \in U} d R\left(n_{R}-n_{\bar{R}}\right)^{2} .
\end{aligned}
$$

Equation (8) must be evaluated together with the condition which guarantees that the total number of agents $N$ is conserved:

$$
N=\int_{R=1}^{R_{\max }} d R n_{R} \equiv \int_{R \in U} d R\left(n_{R}+n_{\bar{R}}\right) .
$$

Equation (8) has a simple interpretation. Since $n_{R}$ and $n \bar{R}$ have opposite sign, they act like two charge-densities of opposite charge which tend to cancel each other out: $n_{R}$ represents a Crowd of like-minded people, while $n_{\bar{R}}$ corresponds to a like-minded Anticrowd who always do exactly the opposite of the Crowd regardless of the specific $\mu(t)$. We have effectively renormalized the charge-densities $n \frac{S}{R}(t) ; \Psi$ and $n \frac{S}{R^{\prime}}(t) ; \Psi$ and their time- and position-dependent two-body interaction $V^{(2)}\left(R, R^{\prime} ; t\right) \equiv a_{R}^{\mu(t)} a_{R^{\prime}}^{\mu(t)}$, to give two identical Crowd-Anticrowd 'quasiparticles' of charge-density $\left(n_{R}-n_{\bar{R}}\right)$ which interact via a time-independent and position-independent interaction term $V_{\mathrm{eff}}^{(2)} \equiv 1$. The different types of Crowd-Anticrowd quasiparticle in Eq. (8) do not interact with each other, i.e. $\left(n_{R}-n \bar{R}\right)$ does not interact with $\left(n_{R^{\prime}}-n \overline{R^{\prime}}\right)$ if $R \neq$ $R^{\prime}$. Interestingly, this situation could not arise in a conventional physical system containing collections of just two types of charge (i.e. positive and negative).

A given numerical simulation will employ a given strategy-allocation matrix (i.e. a given rank-S tensor) $\Psi$. As $R_{\text {max }}$ increases from $1 \rightarrow \infty, \Psi$ tends to become increasingly disordered (i.e. increasingly non-uniform) $[4,9]$ since the ratio of the standard deviation to the mean number of agents holding a particular set of $S$ strategies is equal to $\left[\left(R_{\max }^{S}-1\right) / N\right]^{\frac{1}{2}}$. There are two regimes: (i) A 'high-density' regime where $R_{\max } \ll N$. Here the charge-densities $\left\{n_{R}\right\}$ tend to be large, non-zero values which monotonically decrease with increasing $R$. Hence the set $\left\{n_{R}\right\}$ acts like a smooth function $n(R) \equiv\left\{n_{R}\right\}$. (ii) A 'low-density' regime where $R_{\max } \gg N$. Here $\Psi$ becomes sparse with each element $\Psi_{\mathrm{R}, \mathrm{R}^{\prime}, \mathrm{R}^{\prime \prime}, \ldots}$ reduced to 0 or 1 . The $\left\{n_{R}\right\}$ should therefore be written as 1's or 0's in order to retain the discrete nature of the agents, and yet also satisfy Eq. (9) [4]. Depending on the particular type of game, moving between regimes may or may not produce an observable feature. In the MG, for example, $D_{1}$ does not show an observable feature as $R_{\text {max }}$ increases - however $D_{2}$ does [6]. We leave aside the discussion as to whether this constitutes a true phase-transition [5,9] and instead discuss the explicit analytic expressions for $D_{2}$ which result from Eq. (8). It is easy to show that the mean number of agents using the $X$ th most popular strategy (i.e. after averaging over $\Psi$ ) is [4]:

$$
n_{X}=N\left[\left(1-\frac{(X-1)}{R_{\max }}\right)^{S}-\left(1-\frac{X}{R_{\max }}\right)^{S}\right] .
$$

The increasing non-uniformity in $\Psi$ as $R_{\max }$ increases, means that the popularity-ranking of $\bar{R}$ becomes increasingly independent of the popularity-ranking of $R$. Using Eq. (10) with $S=2$, and averaging over all possible $\bar{R}$ positions in Eq. (8) to reflect the independence of the popularity-rankings for $\bar{R}$ and $R$, we obtain:

$$
D_{2}=\operatorname{Max}\left[\frac{N^{2}}{3 R_{\max }}\left(1-R_{\max }^{-2}\right), \quad N\left(1-\frac{N}{R_{\max }}\right)\right] .
$$

The 'Max' operation ensures that as $R_{\max }$ increases and hence $\left\{n_{R}\right\} \rightarrow 0,1$, Eq. (9) is still satisfied [4]. Equation (11) underestimates $D_{2}$ at small $R_{\max }$ (see Fig. 1) since it assumes that 
the rankings of $\bar{R}$ and $R$ are unrelated, thereby overestimating the Crowd-Anticrowd cancellation. By contrast, an overestimate of $D_{2}$ at small $R_{\max }$ can be obtained by considering the opposite limit whereby $\Psi$ is sufficiently uniform that the popularity and strategy-performance rankings are identical. Hence the strategy with popularity-ranking $X$ in Eq. (10) is anticorrelated to the strategy with popularity-ranking $R_{\max }+1-X$. This leads to a slightly modified first expression in Eq. (11): $\frac{2 N^{2}}{3 R_{\max }}\left(1-R_{\max }^{-2}\right)$. Figure 1 shows that the resulting analytical expressions reproduce the quantitative trends in the standard deviation $D_{2}^{1 / 2}$ observed numerically for all $N$ and $R_{\max }$, and they describe the wide spread in the numerical data observed at small $R_{\max }$.

An important practical implication of the present paper is that the wide range of clusterbased approximation schemes developed in conventional many body-theory, might therefore usefully be extended to capture the dominant correlations in multi-agent competitive populations. Such generalizations will undoubtedly raise interesting issues for the Physics community, concerning the precise manner in which time-averages and configuration-averages should be performed within these traditional approximation schemes.

\section{REFERENCES}

[1] J.L. Casti, Would-be Worlds (Wiley, New York, 1997).

[2] A.C.C. Coolen, The Mathematical Theory of Minority Games (Oxford University Press, 2005); D. Challet, M. Marsili, Y.C. Zhang, Minority Games (Oxford University Press, 2004). See also http://www.unifr.ch/econophysics/minority

[3] A. Soulier and T. Halpin-Healy, Phys. Rev. Lett. 90, 258103 (2003); A. Bru, S. Albertos, J.A. Lopez Garcia-Asenjo, and I. Bru, Phys. Rev. Lett. 92, 238101 (2004); B. Huberman and R. Lukose, Science 277, 535 (1997); B. Arthur, Amer. Econ. Rev. 84, 406 (1994); J.M. Epstein, Proc. Natl. Acad. Sci. 99, 7243 (2002). See also the works of D. Wolpert and K. Tumer at http://ic.arc.nasa.gov

[4] N.F. Johnson and P.M. Hui, cond-mat/0306516 N.F. Johnson, P. Jefferies, P.M. Hui, Financial Market Complexity (Oxford University Press, 2003).

[5] D. Challet and Y.C. Zhang, Physica A 246, 407 (1997); D. Challet, M. Marsili and R. Zecchina, Phys. Rev. Lett. 82, 2203 (1999); J.A.F. Heimel, A.C.C. Coolen and D. Sherrington, Phys. Rev. E 65, 016126 (2001); A. Cavagna, J.P. Garrahan, I. Giardina and D. Sherrington, Phys. Rev. Lett. 83, 4429 (1999).

[6] R. Savit, R. Manuca and R. Riolo, Phys. Rev. Lett. 82, 2203 (1999).

[7] N.F. Johnson, P.M. Hui, R. Jonson and T.S. Lo, Phys. Rev. Lett. 82, 3360 (1999); S. Hod and E. Nakar, Phys. Rev. Lett. 88, 238702 (2002); E. Burgos, H. Ceva, and R.P.J. Perazzo, Phys. Rev. Lett. 91, 189801 (2003); R. D'hulst and G.J. Rodgers, Physica A 270, 514 (1999). These works focus on probabilistic strategies.

[8] N.F. Johnson, M. Hart and P.M. Hui, Physica A 269, 1 (1999); M. Hart, P. Jefferies, N.F. Johnson and P.M. Hui, Physica A 298, 537 (2001); S.C. Choe, P.M. Hui and N.F. Johnson, Phys. Rev. E 70, 055101(R) (2004); M. Hart, P. Jefferies, N.F. Johnson and P.M. Hui, Phys. Rev. E 63, 017102 (2001).

[9] P. Jefferies, M.L. Hart and N.F. Johnson, Phys. Rev. E 65, 016105 (2002).

[10] G.D. Mahan, Many-Particle Physics (Plenum Publishing, New York, 2000) 3rd Edition.

[11] We interchange the order of the $\Psi$ and $t$-averaging over the product of the $n_{R}$ 's. Numerical simulations suggest this is valid for the systems of interest.

[12] T.S. Lo, K.P. Chan, P.M. Hui, N.F. Johnson, Phys. Rev. E 71, 050101(R) (2005). 